\title{
Laparoscopically assisted ilio-inguinal lymph node dissection versus inguinal lymph node dissection in melanoma
}

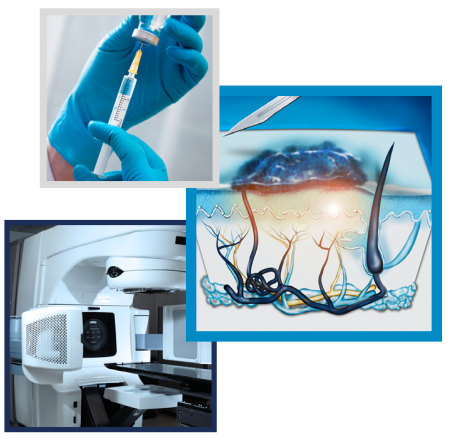

\author{
Enrique Boldo*,1(D), Araceli Mayol' ${ }^{1}$, Rafael Lozoya1, Alba Coret ${ }^{1}$, Diana Escribano ${ }^{1}$, Carlos \\ Fortea ${ }^{1}$, Andres Muñoz ${ }^{1}$, Juan Carlos Pastor ${ }^{1}$ \& Guillermo Perez De Lucia ${ }^{1}$ \\ ${ }^{1}$ Department of Surgery Consorcio Hospitalario Provincial de Castellon, Castellón, Spain \\ *Author for correspondence: eboldo@aecirujanos.es \\ Practice points \\ - Inguinal or ilio-inguinal lymphadenectomy is still needed in melanoma management. \\ - Morbidity of inguinal or ilio-inguinal lymphadenectomy can be reduced with the preservation of greater \\ saphenous vein and the use of minimally invasive therapies. \\ - Other technical details to reduce morbidity of inguinal lymphadenectomy, like transposition of sartorius muscle \\ or use of fibrin seem to be ineffective. \\ - Always re-evaluate your surgical practice and look for new approaches that may minimize postoperative course. \\ - Inguinal microscopic or CT scan/PET-CT negative pelvic inguinal lymph node involvement may be associated to \\ pelvic lymph node involvement. \\ - The need to add pelvic lymphadenectomy to inguinal lymphadenectomy in melanoma with negative clinical \\ pelvic lymph node involvement will hopefully be elucidated by the EAGLE-FM trial.
}

\begin{abstract}
Aim: Morbidity of open inguinal lymphadenectomy (OIL) is high. We use laparoscopy for pelvic time, preservation of the greater saphenous vein and transverse inguinal incisions (laparoscopically assisted ilioinguinal lymphadenectomy, LIIL) to improve postoperative outcomes. Patients \& methods: Retrospective comparison of 14 patients who underwent LIIL and seven patients who underwent OIL. Results: Fourteen LIIL compared with seven OIL showed a statistically significant reduction in morbidity (15.3 vs $75 \%$ ) and hospital stay (7 vs 15.7 days). Pelvic lymph node involvement (27\%) was not detected preoperatively. With a mean follow-up of 36.2 (range: 3-137) months, local recurrence rate was $58.3 \%$ in LIIL and $40 \%$ in OIL. Overall survival was significantly higher in OIL than in LIIL. Conclusion: Compared with OIL, LIIL reduced postoperative complications and hospital stay.
\end{abstract}

First draft submitted: 26 December 2019; Accepted for publication: 12 March 2020; Published online: 21 July 2020

Keywords: comparative effectiveness $\bullet$ hospital stay $\bullet$ lymph node $\bullet$ minimally invasive therapy $\bullet$ oncology $\bullet$ postoperative complications • quality of life $\bullet$ skin (melanoma) • surgery

Following the results of the MSLT-II and DeCOG-SLT trials [1,2], the melanoma clinical guidelines have accepted observation instead of completion lymph node dissection (CLND) in case of microscopic lymph node involvement (positive sentinel lymph node biopsy). However, the melanoma surgeon should still have inguinal lymphadenectomy or ilio-inguinal lymphadenectomy in their armamentarium since approximately between 18 and $26 \%$ of patients with positive sentinel lymph node under observation may develop clinical lymph node involvement $[3,4]$. To this percentage, we must add patients who have clinical/palpable nodal involvement at presentation, which is approximately $38 \%[3]$.

One of the factors that impelled investigators to study the possibility of observation instead of CLND is the morbidity associated to this procedure. Morbidity of inguinal and ilio-inguinal lymphadenectomy has been as high as $52 \%$ of patients in a recent meta-analysis [5]. Therefore, many physicians have introduced technical modifications to reduce morbidity, for example: transposition of the sartorius muscle, use of fibrin, conservation of the greater saphenous vein (GSV) and minimally invasive incisions [5]. Other groups have opted for minimally invasive 


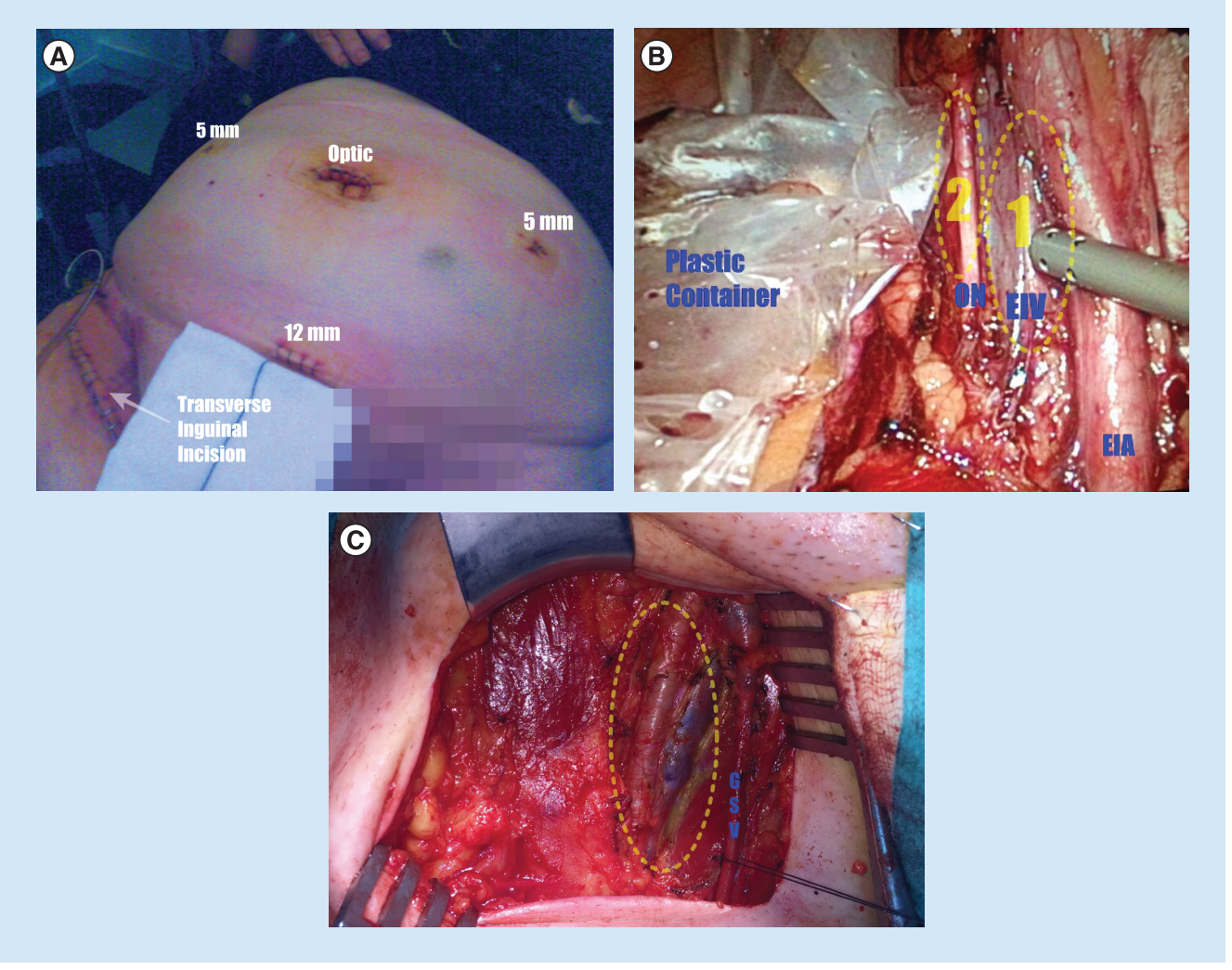

Figure 1. Laparoscopically assisted ilio-inguinal lymphadenectomy procedure. (A) Post operative view of laparoscopically assisted ilio-inguinal lymphadenectomy (LIIL) with trocar distribution for the laparoscopic part and transverse incision for the inguinal part. (B) Laparoscopic part of LIIL, right side. EIA and vein EIV are the anatomical references for medial iliac external nodes (yellow-dotted zone 1); the ON is the anatomical reference for obturator nodes (yellow-dotted zone 2); a plastic container is used for lymph node retrieval. (C) Inguinal part of the LIIL, right side. Transverse inguinal incision permits dissection of lymph nodes from the inguinofemoral content to the apex of the femoral triangle (yellow-dotted circle). The greater saphenous vein has been preserved.

EIA: External iliac artery; EIV: External iliac vein; GSV: Greater saphenous vein; ON: Obturator nerve.

approaches for pelvic dissection and for inguinal dissection [6]. Following this tendency, our group switched from open inguinal lymphadenectomy (OIL) [7] to laparoscopically assisted pelvic lymph node dissection and inguinal lymphadenectomy through transverse incision (LIIL) (instead of the 'lazy S' described by Karakoisis [8]), preserving the GSV whenever possible.

This article compares our results with both techniques, analyzing the current controversies through this comparison.

\section{Patients \& methods}

This is a retrospective study comparing our LIIL case series with a historical OIL case series. In the LIIL technique, four trocars are inserted. One trocar is placed in the umbilicus for optics, and a second 10- to $12-\mathrm{mm}$ trocar is placed centrally just above the pubic bone. The suprapubic trocar generally is disposable to facilitate lymph node extraction. Two 5-mm trocars are placed between the epigastric vessels and the anterior superior iliac spine (Figure 1A). The anatomic borders of lymphadenectomy at this step are the epigastric bundle and the inguinal ligament. The adventitia of the vessels is dissected, and the medial iliac external nodes are retrieved (Figure 1B). All the lymphatic material between the vessels and the pelvic wall is retrieved. Then the lymphatic pad of the obturator fossa is detached from the pelvic wall and collected after further visualization of the obturator nerve (Figure 1B). The limits of dissection are anterior to the pubic bone, medial to the external iliac vessels, inferior to the obturator nerve and posterior to the lumbosacral fossa where the lumbosacral nerve can be identified. The nodes are retrieved in a plastic container. The inguinal part of the LIIL is performed using a transverse incision and involved complete 


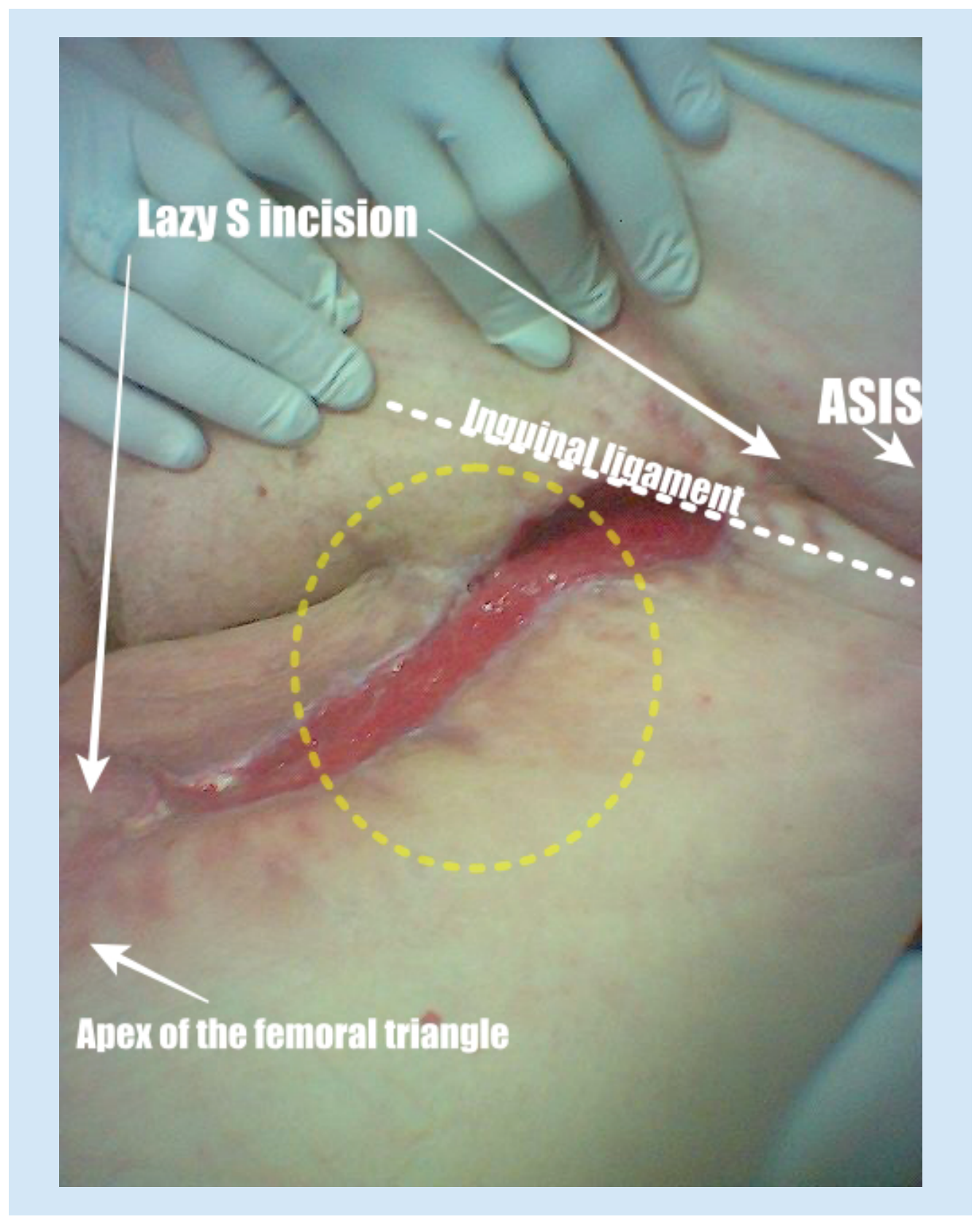

Figure 2. Postoperative view of open inguinal lymphadenectomy, left side. 'Lazy S' inguinal incision (from ASIS to the apex of the femoral triangle) permits complete dissection of lymph nodes from the inguinofemoral content, below the inguinal ligament, to the apex of the femoral triangle (yellow-dotted circle).

ASIS: Anterosuperior iliac spine.

dissection of lymph nodes from the inguinofemoral content to the apex of the femoral triangle (Figure 1C). The GSV is preserved whenever possible.

The OIL was performed via a 'lazy S' inguinal incision (from the anterosuperior iliac spine to the apex of the femoral triangle) and involved complete dissection of lymph nodes from the inguinofemoral content to the apex of the femoral triangle (Figure 2). Sartorius muscle transposition to cover and protect femoral vessels was always performed. The GSV was never preserved. Demographic, oncological and surgical technique data as well as morbidity and hospital stay were collected. The means of the values in the quantitative variables were compared using the Mann-Whitney $U$ test and the qualitative variables the Fisher's exact test (FET). The overall survival curves were calculated by the Kaplan-Meier procedure. 


\begin{tabular}{|c|c|c|c|}
\hline Parameters & LIIL & OIL & Test \\
\hline $\mathrm{N}$ & 14 & 7 & \\
\hline M:F & $8: 6$ & $3: 4$ & \\
\hline AGE & 64.3 & 65.1 & $U M W=34 ; p>0.05$ \\
\hline BMI & 27.5 & 28.4 & $U M W=14 ; p>0.05$ \\
\hline Melanoma T stage, \% & $\begin{array}{l}\text { Tx } 14.2 \\
\text { Tis } 7 \\
\text { T1 } 0 \\
\text { T2 } 21.2 \\
\text { T3 } 14.2 \\
\text { T4 } 42.7\end{array}$ & $\begin{array}{l}\text { Tx } 0 \\
\text { Tis } 0 \\
\text { T1 } 14.2 \\
\text { T2 } 14.2 \\
\text { T3 } 28.5 \\
\text { T4 } 42.8\end{array}$ & \\
\hline Surgical time (min) & 209 & 182 & $U M W=34.5 ; p>0.05$ \\
\hline GSV preservation (\%) & 50 & 0 & FET $=0.046 ; p<005$ \\
\hline Hospital stay (days) & 7 & 15.7 & $U M W=10 ; p<0.05$ \\
\hline Drainage output at discharge (cc) & 233.7 & 52.5 & $U M W=2 ; p<0.05$ \\
\hline Duration of drainage (days) & 16.7 & 13.4 & UMW $=25.5 ; p<0.05$ \\
\hline Postoperative complications (\%) & 15.3 & 75 & $\mathrm{FET}=0.023 ; \mathrm{p}<0.05$ \\
\hline
\end{tabular}

\section{Results}

A total of 14 LIIL were collected and compared with a historical series of seven OIL. Both populations were demographically comparable (Table 1). They were also comparable regarding the use of systemic treatments $(69.2 \%$ of the LIIL and $75 \%$ of the OIL, FET $=1 ; \mathrm{p}>0.05)$. Sixty percent of indications of lymphadenectomy were due to clinical/macroscopic/palpable inguinal lymph node involvement at presentation. The remaining $40 \%$ of lymphadenectomies were indicated as CLND due to microscopic involvement of the sentinel node.

When analyzing the surgical technique, no statistically significant differences were found in the operative time of LIIL and OIL (Table 1). The preservation of the GSV in the LIIL could be achieved in half of the cases.

Given the relevance of the topic in the literature, we also compared the cases in which the GSV was not preserved (50\% LIIL and all the OIL) with those in which the GSV was preserved. Statistically significant differences were found in hospital stay (13 vs 4 days) and in the higher incidence of postoperative complications $(\mathrm{FET}=0.035 ; \mathrm{p}<0.05)$, but not in duration of drainage (15.3 vs 16.3 days).

Regarding postoperative course, the length of hospital stay and incidence of postoperative complications were statistically significantly higher in OIL than LIIL (Table 1). The most frequent postoperative complication of our case series was infection (19\%) followed by cutaneous necrosis, with or without wound breakdown, (14.2\%). About $42.5 \%$ of the LIIL presented lymphedema, this parameter was not recorded in the OIL series.

When comparing path results, a greater number of lymph nodes were obtained in the LIIL compared with the OIL (13.1 vs 8.5$)$ but this difference was not statistically significant (Mann-Whitney $U$ test $=16 ; \mathrm{p}>0.05$ ). The pathological pelvic lymph node involvement was $27 \%$, in all cases with concomitant inguinal lymph node involvement ( $25 \%$ microscopic). Or from another point of view, $11 \%$ of clinically negative groins had pathologically proven pelvic involvement, while this rate increased to $60 \%$ when inguinal lymphadenopathies were palpable. Of note, this pelvic lymph node involvement was not detected preoperatively in any case (half of the cases used CT scan and the other half used PET-CT). The lymph node (LN) ratio (number of nodes affected/number of nodes resected) of both LIIL and OIL was 0.23 .

With a mean follow-up of 36.2 (range: 3-137) months, local recurrence rate was $58.3 \%$ in LIIL and $40 \%$ in OIL, this difference not being statistically significant $(\mathrm{FET}=0.619 ; \mathrm{p}>0.05)$.

Overall survival was significantly higher in the OIL group than in the LIIL group (Figure 3).

\section{Discussion}

In this article, we tried to elucidate if our change in technique for lymphadenectomy, from OIL to LIIL has been beneficial in terms of operative and postoperative outcomes. The absence of differences in surgical times between both techniques (the LIIL comprising the OIL - but using a transverse incision and without sartorius muscle 


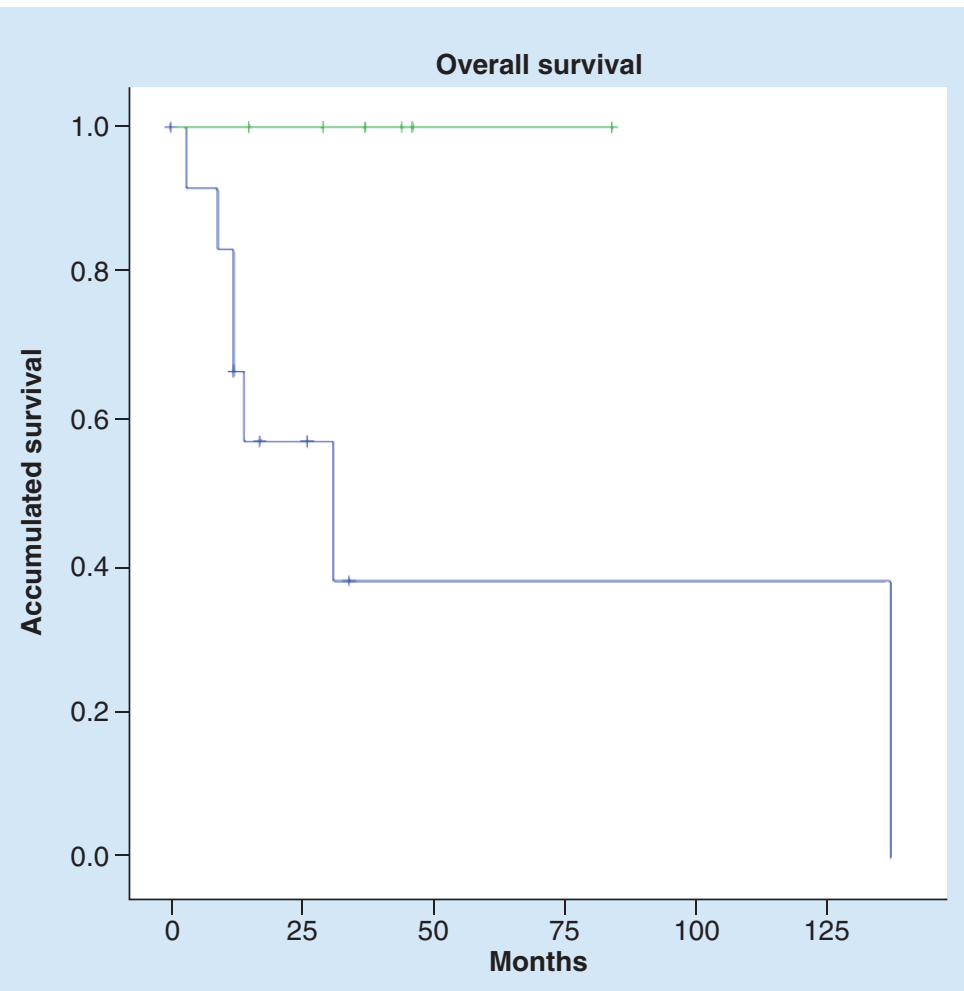

Figure 3. Laparoscopically assisted ilio-inguinal lymphadenectomy and open inguinal lymphadenectomy survival curves. Green: open inguinal lymphadenectomy. Blue: laparoscopicly assisted ilio-inguinal lymphadenectomy.

transposition - plus the laparoscopic pelvic time), can only be explained by the fact that it is a comparison with a historical series. The learning curve (more patients in the OIL historical series) as well as the abandonment of the transposition of the sartorius muscle (with the change to LIIL) could possibly explain the lack of significant differences in surgical time.

In our experience, the preservation of the GSV (Figure 1C) adopted with the LIIL is an effective measure to reduce postoperative complications and hospital stay. The abandonment of the transposition of the sartorius muscle adopted with the LIIL was not associated with a higher incidence of postoperative complications. These findings are in line with the findings of Söderman et al., who described that the use of fibrin doesn't confer benefits [5].

The significant decrease in the incidence of postoperative complications (and therefore in hospital stay) is the main finding of our analysis. This change of technique (with fewer wound complications) allows us to discharge patients with ambulatory drainage management and with a significant earlier drainage removal. Other authors have changed the open approach to videoscopy of not only the pelvic time of lymphadenectomy, but also the inguinal time [6]. The addition of inguinal videoscopy doubled the surgical time. The wound infection rate was $13 \%$ and seroma formation $30 \%$, with no evidence of wound dehiscence [6]. The median hospital stay (7 days) was similar to our series. The lymphedema rate (8\%) was much lower than in our series. These results would suggest that a greater decrease in morbidity can still be achieved (especially in wound dehiscence and lymphedema), if a completely videoscopic approach is adopted. Table 2 presents are review of the inguinal videoscopic procedure. After the initial experiences published with small patient numbers, some more robust case series can be analyzed [7]. The rate of complications remains high despite the use of videoscopy. Lymphedema rate is $54 \%$ [7], even higher than in our experience with LIIL. The most remarkable outcome is that, generally, mean hospital stay has been reduced to 1 day $[7,9,10]$. Finally, other authors have preferred to undertake a videoscopic inguinal approach followed by open iliac lymph node dissection via a separate skin incision [11]. Compared with LIIL, mean operative time was halved, but complication rate doubled (37.5\%). The rate of lymphedema $(44.8 \%)$ - mild and moderate - was slightly higher than in LIIL. 


\begin{tabular}{|c|c|c|c|c|c|c|}
\hline Study (year) & Technique & $\mathbf{n}$ & $\begin{array}{l}\text { Operative time (MEAN) } \\
\text { (min) }\end{array}$ & Complication rate, \% & $\begin{array}{l}\text { Hospital stay (MEAN) } \\
\text { (days) }\end{array}$ & Ref. \\
\hline Jakub (2017) & MIIL & 77 & $\mathrm{~N} / \mathrm{A}$ & 71 & 1 & [7] \\
\hline Delman (2010) & MIIL & 5 & 180 & 40 & 1 & [9] \\
\hline Ichimiya (2013) & MIIL & 5 & 163 & 20 & $N / A$ & [12] \\
\hline Abbott (2013) & MIIL & 13 & 245 & 0 & 1 & [10] \\
\hline
\end{tabular}

MIIL: Minimally invasive inguinal lymph node dissection; N/A: Not available.

The median number of lymph nodes obtained in both the LIIL and the OIL was higher than the 90th percentile of results from reference centers in the treatment of melanoma [13]. This quality assessment of lymphadenectomies was suggested by Spillane et al. [14], in the absence of an established lymph node cut-off number that should contain a lymphadenectomy. Obviously, we obtained a greater number of lymph nodes in the LIIL, although this difference with the OIL did not reach statistical significance. In addition, BMI was not a confounding factor [15], as there was not a significant difference between the two groups.

It is necessary to express that with an analyses of 21 patients, our patient numbers are too low to draw any conclusion regarding oncological outcomes. Nevertheless, we would like to present to the readership, our reflections after contrasting our discrete experience with the literature, while awaiting the results of randomized trials.

Our local recurrence rate was higher than in the literature, which varies from one digit to $35 \%[6,16,17]$. The fact that $60 \%$ of the patients in our series had macroscopic inguinal involvement, with a LN ratio category B (10-25\%) and more than $40 \%$ of T $4 \mathrm{~s}$, reflect a locally advanced melanoma case series, with an expected worse local recurrence rate. It has been proposed that there are differences between microscopic and macroscopic inguinal involvement in relation to tumor biology, incidence of lymph node involvement and recurrence pattern or survival [16]. Perhaps our high incidence of macroscopic node involvement justifies the higher incidence of local recurrence and even the paradox of a worse incidence of local recurrence with more extensive surgery.

Another finding from our series has been better survival following OIL compared with LIIL. First, it is worth highlighting the methodological problem that Kaplan-Meier survival curves lose reliability with an $\mathrm{N}$ below 30. Nevertheless, other authors found better survival from OIL to LIIL; however, unlike our results, statistical significance was not demonstrated [16,18]. Contrary to the findings of Rossi et al. [15], the greater number of resected nodes has not been associated with a better prognosis of LIIL (Figure 3). Rossi et al. also linked low values $(<0.1)$ in the LN ratio to a better prognosis [15]. We couldn't verify this fact in our small series, where the OIL LN ratio should have theoretically been lower than in the LIIL. The improvement in the prognosis with more extensive lymphadenectomies seems to be limited to patients with microscopic nodal disease [15].

The EAGLE-FM trial, currently recruiting, is intended to corroborate if the extent of lymphadenectomy (adding pelvic lymphadenectomy to inguinal lymphadenectomy) influences local control and patient survival [16]. It has been advocated that pelvic lymphadenectomy should be added to inguinal lymphadenectomy when there is clinically gross involvement of the groin, when there are clinically detectable deep lymph nodes, when Cloquet's node is histologically positive or when pelvic CT scan demonstrates pelvic lymphadenopathy [17]. If we had followed the indication of performing pelvic lymph node dissection only if inguinal lymph node involvement is macroscopic, one quarter of our patients with pathologically proven pelvic involvement would have not received lymphadenectomy of this territory. On the other hand, the performance of pelvic lymphadenectomy only in our cases of positive imaging tests would not have been a successful approach either. Even with the use of CT scan and PET-CT, a third of our patients with negative imaging tests did have pathologically proven pelvic involvement.

In conclusion, the change from OIL to LIIL has allowed us to reduce postoperative complications and hospital stay. In our limited experience, preservation of GSV is an effective measure to reduce postoperative complications.

\section{Future perspective}

New developments in biological therapy for melanoma will probably leave lymphadenectomy only for relapsed or resistant lymph node disease. Nevertheless, surgeons must always incorporate novel approaches (like minimally invasive techniques) to improve results. 


\section{Author contributions}

E Boldo: conceptualization, data curation, formal analysis, funding acquisition, investigation, methodology, writing; A Mayol: conceptualization, data curation, formal analysis, investigation, methodology, writing; R Lozoya: conceptualization, data curation, formal analysis, investigation, methodology, writing; A Coret: data curation, formal analysis, writing; D Escribano: data curation, formal analysis, writing; C Fortea: data curation, formal analysis, writing; A Muñoz: data curation, formal analysis, writing; JC Pastor: data curation, formal analysis, writing; GPD Lucia: data curation, formal analysis, writing.

\section{Financial \& competing interests disclosure}

The authors have no relevant affiliations or financial involvement with any organization or entity with a financial interest in or financial conflict with the subject matter or materials discussed in the manuscript. This includes employment, consultancies, honoraria, stock ownership or options, expert testimony, grants or patents received or pending, or royalties.

No writing assistance was utilized in the production of this manuscript.

\section{Ethical conduct of research}

The authors certify that appropriate institutional review board approval has been obtained and that informed consent has been obtained from the participants involved, specifically for graphic material taken for academic or scientific purposes.

\section{Open access}

This work is licensed under the Attribution-NonCommercial-NoDerivatives 4.0 Unported License. To view a copy of this license, visit http://creativecommons.org/licenses/by-nc-nd/4.0/

\section{References}

Papers of special note have been highlighted as: $\bullet$ of interest; $\bullet \bullet$ of considerable interest

1. Morton DL. Overview and update of the Phase III multicenter selective lymphadenectomy trials (MSLT-I and MSLT-II) in melanoma. Clin. Exp. Metastasis 29(7), 699-706 (2012).

\section{-. Pivotal study, practice-changing paper.}

2. Leiter U, Stadler R, Mauch C et al. Complete lymph node dissection versus no dissection in patients with sentinel lymph node biopsy positive melanoma (DeCOG-SLT): a multicenter, randomized, Phase III trial. Lancet Oncol. 17(6), 757-767 (2016).

3. Callender GG, McMasters KM. Early versus delayed complete lymphadenectomy in melanoma: insight from MSLT I. Ann. Surg. Oncol. 18(2), 306-308 (2011).

4. Faries MB, Thompson JF, Cochran AJ et al. Completion dissection or observation for sentinel-node metastasis in melanoma. N. Engl. J. Med. 376, 2211-2222 (2017).

5. Söderman M, Thomsen JB, Sørensen JA. Complications following inguinal and ilioinguinal lymphadenectomies: a meta-analysis. J. Plast. Surg. Hand Surg. 50(6), 315-320 (2016).

- Best data to identify the problem of morbidity after ilio-inguinal lymph node dissection.

6. Sommariva A, Pasquali S, Cona C et al. Videoscopic ilioinguinal lymphadenectomy for groin lymph node metastases from melanoma. Br. J. Surg. 103(8), 1026-1032 (2016).

- Important case series that show the advantages of complete endoscopic approach.

7. Jakub JW, Terando AM, Sarnaik A et al. Safety and feasibility of minimally invasive inguinal lymph node dissection in patients with melanoma (SAFE-MILND): report of a prospective multi-institutional trial. Ann. Surg. 265(1), 192-196 (2017).

8. Karakousis CP, Emrich LJ, Rao U. Groin dissection in malignant melanoma. Am. J. Surg. 152(5), 491-495 (1986).

9. Delman KA, Kooby DA, Ogan K, Hsiao W, Master V. Feasibility of a novel approach to inguinal lymphadenectomy: minimally invasive groin dissection for melanoma. Ann. Surg. Oncol. 17(3), 731-737 (2010).

10. Abbott AM, Grotz TE, Rueth NM et al. Minimally invasive inguinal lymph node dissection (MILND) for melanoma: experience from two academic centers. Ann. Surg. Oncol. 20(1), 340-345 (2013).

11. Vrielink OM, Faut M, Deckers EA, van Leeuwen BL, Been LB. Evaluation of the videoscopic inguinal lymphadenectomy in melanoma patients. Eur. J. Surg. Oncol. 45(9), 1712-1716 (2019).

12. Ichimiya M, Goishi K, Muto M. Endoscopic groin lymph node dissection as a preferable technique for malignant skin neoplasms. J. Plast. Surg. Hand Surg. 47(4), 248-251 (2013).

13. Berger-Richardson D, Cordeiro E, Ernjakovic M, Easson AM. Lymph node retrieval rates in melanoma: a quality assessment parameter. Curr. Oncol. 24(4), e323-e327 (2017).

14. Spillane AJ, Haydu L, McMillan W, Stretch JR, Thompson JF. Quality assurance parameters and predictors of outcome for ilioinguinal and inguinal dissection in a contemporary melanoma patient population. Ann. Surg. Oncol. 18(9), 2521-2528 (2011). 


\section{-• Quality assessment for lymphadenectomies.}

15. Rossi CR, Mozzillo N, Maurichi A et al. Number of excised lymph nodes as a quality assurance measure for lymphadenectomy in melanoma. JAMA Surg. 149(7), 700-706 (2014).

16. Verver D, Madu MF, Oude Ophuis CMC et al. Optimal extent of completion lymphadenectomy for patients with melanoma and a positive sentinel node in the groin. Br. J. Surg. 105(1), 96-105 (2018).

17. van der Ploeg AP, van Akkooi AC, Schmitz PI et al. Therapeutic surgical management of palpable melanoma groin metastases: superficial or combined superficial and deep groin lymph node dissection. Ann. Surg. Oncol. 18(12), 3300-3308 (2011).

18. Egger ME, Brown RE, Roach BA et al. Addition of an iliac/obturator lymph node dissection does not improve nodal recurrence or survival in melanoma. J. Am. Coll. Surg. 219(1), 101-108 (2014). 